\section{EDUCATION}

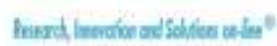

Electronic Journal of Research

in Educational Psychology

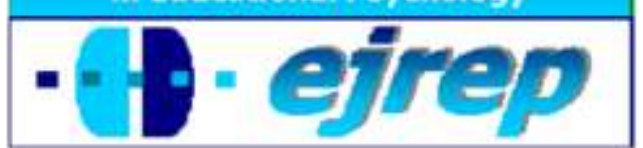

\title{
Significado de la educación sexual en un contexto de diversidad de Venezuela
}

\author{
Denyz Luz Molina ${ }^{1}$, Irsia Rosa Torrivilla ${ }^{2}$ \\ y Yolimar Gregoria Sánchez ${ }^{3}$
}
${ }^{1}$ Universidad Nacional Experimental de los Llanos Occidentales "Ezequiel Zamora". Barinas
${ }^{2}$ Grupo Escolar Guarico. Barinas
${ }^{3}$ Universidad Nacional Experimental de los Llanos Occidentales "Ezequiel Zamora". Barinas

\section{Venezuela}




\section{Resumen}

Introducción. En esta investigación se pretende presentar una aproximación a la construcción del significado que le confieren los docentes, alumnos y familias a la educación sexual en los centros escolares y el aula. El mismo se aborda en un contexto de diversidad en Venezuela, desde diversas perspectivas teóricas de la educación sexual, lo que ha ayudado a la contrastación con los conceptos que manejan los docentes y familias, llevándonos a determinar categorías que ayudaron a perfilar un concepto de educación sexual integral.

Método. Mediante la investigación descriptiva-etnográfica se indaga en una perspectiva política, normativa y práctica sobre líneas de acción en materia de educación sexual que se manejan a nivel de los currículos de educación primaria y secundaria. Igualmente, se analizan las estrategias que los docentes, los padres y la familia utilizan para promover la educación sexual a nivel de los escolares y su incidencia en los padres y comunidad. El estudio se abordó en una muestra representada por 20 alumnos, 20 docentes y 15 familias pertenecientes a dos centros escolares que imparten educación primaria y secundaria, ubicada en el Municipio Barinas, del Estado Barinas (Venezuela). La información se acopió a través de registros diarios, entrevistas y cuestionarios con preguntas abiertas. Los datos fueron recogidos a lo largo de un año escolar mediante el uso de las técnicas e instrumentos que han facilitado la organización, categorización y análisis de la información, con apoyo de las herramientas que brinda la estadística descriptiva.

Resultados. El concepto de educación sexual que manejan los escolares, docentes y familias esta matizado, por su experiencia, vivencias, cultura y tradiciones, lo que ha llevado a que esencialmente el significado que se le confiere es evidentemente particular, determinista y centrado en una concepción de la sexualidad con finalidad exclusivamente reproductiva. La escuela fundamentalmente promueve la información más que la formación en educación sexual, las acciones están centradas al cumplimiento de determinados objetivos curriculares, en la mayoría de los casos de dominio cognitivo, y la intervención de los padres es limitada o casi nula.

Discusión y Conclusiones. De los análisis se derivan algunas premisas que nos ayudarían al diseño de planes y programas de intervención en educación sexual integral desde los primeros años de vida, que involucre al escolar y a su entorno inmediato, en el desarrollo de planes, 
programas y proyectos educativos, para que se apropien en colectivo del concepto de integralidad que representa la educación sexual. También se plantea a los centros escolares la necesidad de asumir previo a cada año escolar un programa de intervención en educación sexual integral donde se valoren las experiencias, vivencias, conocimientos e información recibida en el contexto escolar, familiar y comunitario, con el fin de apropiarse estrategias de orientación sexual con mayor pertinencia social.

Palabras Clave: Construcción de significados, educación sexual, integralidad, diversidad. 


\title{
Meaning of sexual education in a context of diversity of Venezuela
}

\begin{abstract}
Introduction. In this research intends to present an approach to the construction of meaning that give teachers, students and families to sex education in schools and the classroom. The same is addressed in a context of diversity in Venezuela, in effect set out different theoretical perspectives of sex education, which has helped the contrast with the concept of what teachers and families, leading us to identify categories that helped shape a concept of comprehensive sex education.
\end{abstract}

Method. Through descriptive-ethnographic research explores the political perspective, policy and practice on courses of action in sex education is handled at the level of the curricula of primary and secondary education. Also analyzes the strategies that teachers, parents and the family used to promote sex education of schoolchildren and their impact on parents and community. The study focused on a sample represented by 20 students, 20 teachers and 15 families belonging to two schools that offer primary and secondary education, located in the town Barinas, Barinas State (Venezuela). The information was collected through diaries, interviews and questionnaires with open questions. Data were collected over a school year using the techniques and instruments have facilitated the organization, categorization and analysis of information, with support from the tools provided by descriptive statistics. Results. The concept of sex education that manage schools, teachers and families is colored by his experience, experiences, culture and traditions, which has led to essentially the meaning given to it is obviously particular to identify and focus on a conception of sexuality with reproductive purposes only. The school primarily promotes information that training in sexual education, the actions are focused on the fulfillment of curricular objectives, in most cases cognitive domain, and parental involvement is limited or almost nil.

Discussion and Conclusions. From the analysis leads to some assumptions that help us to design intervention plans and programs in comprehensive sex education from the earliest years of life, involving the school and its immediate environment, developing plans, programs and educational projects for collectively take ownership of the concept of wholeness that represents the sexual education. It also raises the schools need to take before each school year 
a program of comprehensive sex education intervention which valued the experience, experience, knowledge and information received in the school, family and community, in order to appropriate sexual orientation strategies with more social.

Keywords: Construction of meaning, sexual education, integrity, diversity. 


\section{Introducción}

En el presente estudio se analiza el significado que le otorgan los docentes, escolares y familia a la educación sexual en los centros escolares y el aula, tema de indagación que se plantea dada la necesidad de abordar desde los primeros años de vida del niño, niña y adolescente la educación sexual desde el punto de vista de la integralidad. El mismo, tiene su ámbito de investigación en el contexto venezolano, siendo debatido con escasa profundidad y limitado tratamiento a nivel del contexto familiar y comunitario por los centros escolares mediante los proyectos educativos y como estrategia de formación permanente.

Tal como lo expresa Flores, Lugo y Moya (2009) en estudio de los conocimientos generales sobre sexualidad en adolescentes del área urbana de la ciudad de Punto FijoVenezuela, la mayoría de los adolescentes afirman haber recibido Educación Sexual (84,48\%) de regular a mala calidad comprobándose por los escasos conocimientos que tienen sobre tópicos como funcionamiento de su aparato reproductor y el del sexo opuesto, uso de métodos anticonceptivos, modo de transmisión de enfermedades, concluyéndose que sigue siendo muy tímida y pudorosa la educación sexual impartida por padres y docentes, lo cual lleva a que sea influenciada por otras fuentes poco idóneas tales como la televisión y revistas, además de reflejarnos una vez más las grandes dudas y ansiedad que expresan los adolecentes con respecto a temas de sexualidad.

El citado estudio refiere además, entre sus recomendaciones que la educación sexual debe estar destinada a los padres para que puedan trasladar esos conocimientos con naturalidad e idoneidad a sus hijos. La ley no permite a un menor de edad asumir la responsabilidad de contraer matrimonio sin la autorización de sus padres. Esto supone que tampoco es responsable para tener relaciones sexuales ocasionales o estables.

La educación sexual, a juicio de Molina (2009), “implica ir más allá de un abordaje de información, tiene que asumirse desde la perspectiva de la atención a la diversidad, del conocer, respetar y valorar las diferencias individuales y culturales de los alumnos y evitar cualquier tipo de discriminación"; de allí que, la educación sexual sea parte esencial en la formación de la persona, de su autoestima, puesto que permite su integración a la vida social del contexto donde ésta se desenvuelve. Además, podría interpretarse que la diversidad orienta las acciones hacia ver y atender a todo el grupo bajo el principio de integración, 
normalización y equidad, en el que se acepte, valore y respete las diferencias individuales y se valore la educación sexual desde los primeros años de vida del niño.

Así mismo, se considera que la atención a la diversidad es similar a preocuparse por las medidas que puedan compensar las desigualdades y atenuar de alguna manera las desventajas que unos estudiantes experimentan en relación con el acceso, la permanencia y la prosecución dentro del sistema educativo; al igual que, sirve como medio para eliminar las barreras que limiten el aprendizaje y la integración participativa en igualdad de oportunidades al currículo, a las instalaciones físicas y a la vida educativa (Echeita, 2005, p.7).

Por otra parte, Romero \& Lauretti (2006, p. 348) explican que la integración "es un proceso continuo y progresivo cuya finalidad es incorporar al individuo a la sociedad y constituye uno de los fenómenos de mayor trascendencia en la educación"; igualmente, explican que se refiere a la posibilidad que tienen las personas de poder acceder a todos los medios de información en beneficios de su propio desarrollo personal, social, profesional, vocacional y escolar.

En el marco de esta diversidad, todos los educandos tienen derecho a recibir educación sexual integral en los establecimientos educativos públicos, de gestión estatal y privada. Entiéndase como educación sexual integral la que articula aspectos biológicos, psicológicos, sociales, afectivos y éticos. La educación sexual más que un derecho de los niños, niñas y adolescentes es un deber de la familia y de los centros educativos. La educación sexual constituye un proceso formativo, dinámico y permanente, que promueve el desarrollo integral del ser humano, contribuyendo así al desarrollo social de su comunidad.

Desde esta perspectiva, la educación sexual mejora las condiciones de vida de niños, niñas y adolescentes, previene problemas de salud psicofísica, y promueve el cumplimiento de los derechos humanos, fomentando la igualdad entre varones y mujeres, así como, la convivencia familiar y social. La educación sexual promueve la internalización de valores relacionados con el papel de varones y mujeres, sin estereotipos, en un marco de equidad. Además promueve el cuidado propio y el de las demás personas, fomenta la equidad de derechos y facilita el desarrollo de actitudes positivas en torno a la sexualidad. La educación sexual constituye un proceso intencional, constante y transversal, que debe darse a lo largo de todo la vida del sujeto. 
La educación sexual se inicia desde que nacemos a partir de los valores y pautas culturales y de conducta que nos transmiten en nuestra familia y en todos los ámbitos de socialización primaria. Luego esta educación sexual se amplía a la que recibimos en la escuela (educación sexual formal) y a la que incorporamos a través de los medios de comunicación, la web, la publicidad y otros medios de comunicación.

Es necesario señalar que si no existe educación sexual formal en la escuela, los niños y niñas, igual están expuestos a las otras formas informales de educación sexual. En estos casos la información es muy variable, en general transmite nociones contradictorias que confunden a niños y niñas. Cuando se objeta la educación sexual formal porque se cree que introducirá nociones que pueden promover las relaciones sexuales precoces, no se percibe que esto se recibe a través de la educación informal y que la educación sexual formal permitiría que los adolescentes tengan un ámbito en el que puedan reflexionar además de informarse correctamente.

La reflexión y análisis de la adecuada información les permite a los alumnas y alumnos tener elementos para no incorporar lo que desde los medios y otros ámbitos "les venden" y que no los ayuda al ejercicio libre, saludable y placentero de su sexualidad. Otro argumento muy escuchado en contra de la educación sexual formal es que pretende reemplazar a los padres. Esto es falaz, ya que la escuela en este como en los otros temas, no reemplaza a los padres pero si los complementa. De lo contrario, los padres educarían a sus hijos en su casa enseñándoles a escribir, a leer, a sumar y demás.

Las escuelas públicas han incluido en su currículum la educación sexual como una parte regular de la formación por décadas, las controversias son abundantes cuando se trata con este tipo de asuntos delicados y hay en el aire muchas ventajas y desventajas acerca de la educación sexual que se viene enseñando en clases, pero muy pocos padres están en desacuerdo.

Los resultados de las encuestas recientes han demostrado que menos del $7 \%$ de la población no cree que la educación sexual debe ser un requisito en las escuelas. Esto quiere decir que hay un $93 \%$ a favor de que esta materia esté en el currículum regular de las escuelas. No obstante, hay pros y contras que los padres deben considerar cuando se enfrentan a las clases de educación sexual, ya que esta no es una materia como matemática o lectura, en 
general, los padres necesitan estar atentos de su propia familia, sus creencias religiosas y los valores, además de conocer el intelecto de sus hijos y sus niveles de madurez.

Otro de los aspectos en contra, es el hecho que con frecuencia en los centros escolares la educación sexual tiende a la discriminación de género en la información sexual que se promueve, tal es el caso, de las estrategias dirigidas a enseñar a los niños y niñas la función de los órganos reproductores, pareciera que la educación sexual la reducen a la funcionalidad de los órganos sexuales. Los escolares podrán aprender los términos correctos del sistema reproductivo, las enfermedades de transmisión sexual y los métodos anticonceptivos, antes que aprender el lenguaje que se emplea en la calle para estos mismos términos. La educación sexual puede ir en contra de la moral individual o de las creencias religiosas de los escolares y de sus familias.

Los mitos que envuelven al acto sexual pueden quedar aclarados, como por ejemplo, el mito de que en la primera vez no puedes quedar embarazada. Papalía (2006) muestra que muchos adolescentes se vuelven sexualmente activos antes de la inclusión de las clases de educación sexual. La participación temprana de estas clases ayuda a los estudiantes a mantenerse en abstinencia o al menos a ser más responsables de sus decisiones como activos sexualmente. Una apropiada educación sexual puede tener un buen impacto previniendo problemas sexuales en la adultez. Por otro lado, las desventajas de la educación sexual son: los estudiantes pueden tener vergüenza o llegar a excitarse por los tópicos que se tratan en clase, lo que podría derivar en un revuelo en clase si los alumnos se prestan para hacer comentarios inapropiados; la mayoría de las clases de educación sexual se suelen dar como un pequeño intervalo durante una clase de educación física o de salud, por lo cual no son lo suficientemente efectivas para temas de esa importancia; por último, la educación sexual puede ir en contra de la moral individual o de las creencias religiosas de los escolares y de sus familias. Para Hiriart (2000), los adolescentes que reciben una educación sexual adecuada son lo mejor preparados para enfrentar las ansiedades e inquietudes propias de esa edad y para aprender a ejercer una sexualidad sana, madura, gozosa y responsable.

Entre otros efectos contraproducentes, la falta de información, los mitos, los prejuicios o el silencio timorato en torno de la sexualidad propician las relaciones desprotegidas, los embarazos no deseados y las enfermedades de transmisión sexual, además de volver más vulnerables a violaciones y abusos a jóvenes e infantes. 
Conscientes de ello, diversas organizaciones han pugnado por que en las escuelas se imparta educación sexual al menos desde la pubertad. Afortunadamente, hoy en día se cuenta con la voluntad política necesaria para responder a esta legítima demanda de la sociedad y se vive un momento propicio para fortalecer y consolidar una nueva cultura alrededor de los derechos sexuales y reproductivos de la población. En esta coyuntura, se ofrece aquí al docente un valioso instrumento que le ayudará a encontrar la mejor manera de abordar en el salón de clases diversos temas relacionados con la sexualidad y con la psicología del adolescente, y a orientar a sus alumnos en esta etapa de cambio y descubrimiento.

Jaímes (1983),expresa; "la sexualidad es un componente de la personalidad humana que atañe tanto a su composición biológica, emocional y social... realidad física y mental en cada ser humano y una fuerza o potencialidad para ser ejercida...puede ser fuente de inmenso placer pero también fuente de graves trastornos de la vida personal y social del individuo...puede ser un vehículo para la comunicación pero puede ser también un instrumento de explotación... implica, por consiguiente, tanto un don de la naturaleza como una responsabilidad del ser humano"(p.23).

Para Clement (2007) "el enfoque integral es un acercamiento eminentemente humanista que mira al hombre y a la mujer en toda su dimensión esencial y existencial, que acepta la realidad presente, pero también cree en su capacidad de perfeccionamiento...conlleva necesariamente a una toma de conciencia del estado de deshumanización imperante en la realidad actual y un compromiso con la búsqueda de la humanización como proceso permanente y dinámico”. (p.45)

La integralidad tiene que ver con el desarrollo inherente a la persona, su autoestima, tal como lo expresa De Luca (2009), el hombre debe tener la capacidad de gobernarse así mismo, donde el primer requisito de una persona autónoma lo constituye la decisión por parte del ser humano, de asumir directamente la responsabilidad de su propia vida y no dejar que otros decidan en su lugar. (p.907)

Desde la misma perspectiva en el documento: lineamientos para una educación sexual integral (2008):’La Educación Sexual Integral es una acción formativa presente en todo el proceso educativo, que promueve y fortalece el desarrollo sexual saludable en las y los estudiantes, propiciando su ejercicio pleno y responsable, contribuyendo al desarrollo de 
factores protectores que los preparen para enfrentar las situaciones de riesgo que se le presenten a lo largo de toda la vida.’'(p.12)

La condición humana es muy compleja, por lo que para comprenderla recurrimos en oportunidades a ver aspectos específicos como la sexualidad, y a pesar de ello no es posible tener un conocimiento certero, no la podemos aislar de infinidad de factores que confluyen para que tenga lugar. Ahora bien, la sexualidad se entiende en esta propuesta como la forma peculiar de sentirse y vivirse como uno de los sexos, se relaciona con factores biológicos, sociales, culturales, espirituales, psicológicos, económicos y éticos. En este sentido, la educación sexual integral forma parte del proceso formativo y tiene como objetivo desarrollar en las y los alumnos, competencias para el ejercicio saludable, placentero y responsable de su sexualidad.

De allí que, el tema de la educación sexual integral haya sido discutido por diversas organizaciones mundiales, entre las cuales se encuentra la Organización de las Naciones Unidas para la Educación, la Ciencia y la Cultura (UNESCO, 2000) que en el marco de los programas "Educación para todos” realizó en Dakar-Senegal, el Foro Mundial de Educación, donde queda ratificada la preocupación de las diferentes naciones, sobre el derecho que tienen las personas a recibir educación sexual integral.

Estas declaraciones e informes, suscritos por estas organizaciones internacionales, deben ser considerados en todos los sectores de la sociedad puesto que: reafirman el compromiso con la educación para los niños, jóvenes y adultos, en la educación regular o común; y consideran que la diversidad, la valoración de la diferencia, el respeto por la dignidad y la igualdad de todos los seres humanos y la educación sexual integral son principios inseparables de la educación primaria.

De lo anterior, se desprende que el mayor reto de la educación formal mediante la operacionalización de los programas de formación es la integración de la educación sexual integral considerando el entorno comunitario, familiar y escolar.

\section{Objetivos de la investigación}


En consecuencia, mediante la indagación y exploración epistemológica y ontológica de los objetivos que se configuran seguidamente se definirían lineamientos que se integren en los proyectos educativos con el fin de promover una educación sexual integral:

1) Analizar las estrategias de promoción de la educación sexual en los centros escolares y el aula.

2) Determinar el significado que le otorgan los docentes, alumnos y comunidad en general a la educación sexual en el marco de la diversidad.

3) Categorizar el significado que le otorgan los docentes, alumnos y comunidad en general a la educación sexual en el marco de la diversidad.

En Venezuela, específicamente, en el Sub-Sistema de Educación Primaria son escasos los trabajos de investigación que tengan como objetivos el análisis del significado de la educación sexual en la educación primaria y secundaria bajo un contexto de atención a la diversidad: al igual que, la indagación sobre las políticas, programas, servicios y condiciones que se aplican para promover la educación sexual. Se pretende mediante la investigación en curso avanzar en un primer momento en un diagnóstico de necesidades de educación sexual, con el fin de configurar una aproximación a los lineamientos para promover en todos los espacios formativos escolares y extraescolares la educación sexual integral y contextualizada a la dinámica de los escolares en los centros educativos venezolanos.

Estos planteamientos servirían para la apropiación de un nuevo concepto de educación sexual desde el punto de vista formativo y nos llevaría a la resignificación del mismo desde la perspectiva del reconocimiento del sujeto, de su cuerpo y de sus sentimientos como base del trabajo pedagógico. Educar en sexualidad es, por tanto, una forma de apreciar que la vida sucede en un cuerpo y que, como seres humanos, podemos también entender, analizar y cuidar lo que sucede con nuestros cuerpos, como parte del desarrollo integral de nuestra ciudadanía y nuestras relaciones. De tal modo, educar en sexualidad implica tanto ofrecer conocimientos para la prevención de embarazos no deseados e infecciones de transmisión sexual, como formar en valores, sentimientos y actitudes positivas frente a la sexualidad.

Así mismo, la propuesta de lineamientos educativos para el significado de integralidad en la educación sexual, permitiría generar nuevas estrategias que garanticen a los estudiantes que cursan estudios en centros escolares pertenecientes al Ministerio de Educación en los subprogramas de educación primaria y secundaria una atención educativa integral, bajo el 
principio de integración, inclusión y de atención a la diversidad que encierra el concepto de integralidad. Destacando que los centros educativos como gestores y mediadores de aprendizajes deben facilitar, orientar el proceso, respetar, aceptar las necesidades, intereses, potencialidades y ritmo del aprendizaje de sus estudiantes.

En este sentido la educación integral según Molina \& De Luca (2009), “es aquella que se propone como objetivo la formación de la persona en orden a su fin último, que las búsqueda de la libertad la verdad y el bien de las sociedades de las que el hombre es miembro y en cuyas responsabilidades participara cuando llegue a ser adulto.’(p.1453)

Finalmente, la investigación serviría como referente a todos los centros educativos pertenecientes a los subprogramas primarios y secundarios puesto que aportaría cambios significativos en el concepto, significado y actitudes que se le confieren a la educación sexual en los centros escolares y el aula. Así como la promoción de un enfoque integral comunitario hacia la familia y comunidad en general.

El enfoque de la integralidad de la educación sexual debe abarcar las siguientes dimensiones de análisis: el desarrollo psicofísico, la vida de relación, la salud, la cultura y la espiritualidad y se manifiesta de manera diferente en las distintas personas y etapas de la vida; la valoración de la comunicación y el amor como componentes centrales de la sexualidad; el reconocimiento y la valoración de la responsabilidad y el derecho a la intimidad como elementos indispensables en los comportamientos sexuales; el respeto a la diversidad de valores en sexualidad; el reconocimiento y la valoración del derecho de las niñas y niños, adolescentes y jóvenes a ser especialmente amados/as, protegidos/as y cuidados/as; y el reconocimiento y valoración de las familias como ámbito de cuidado y formación de los niños/as, adolescentes y jóvenes.

Seguidamente se presenta en el la fig. 1 los elementos teóricos implícativos que nos ayudarían a la construcción del significado de la educación sexual integral. 


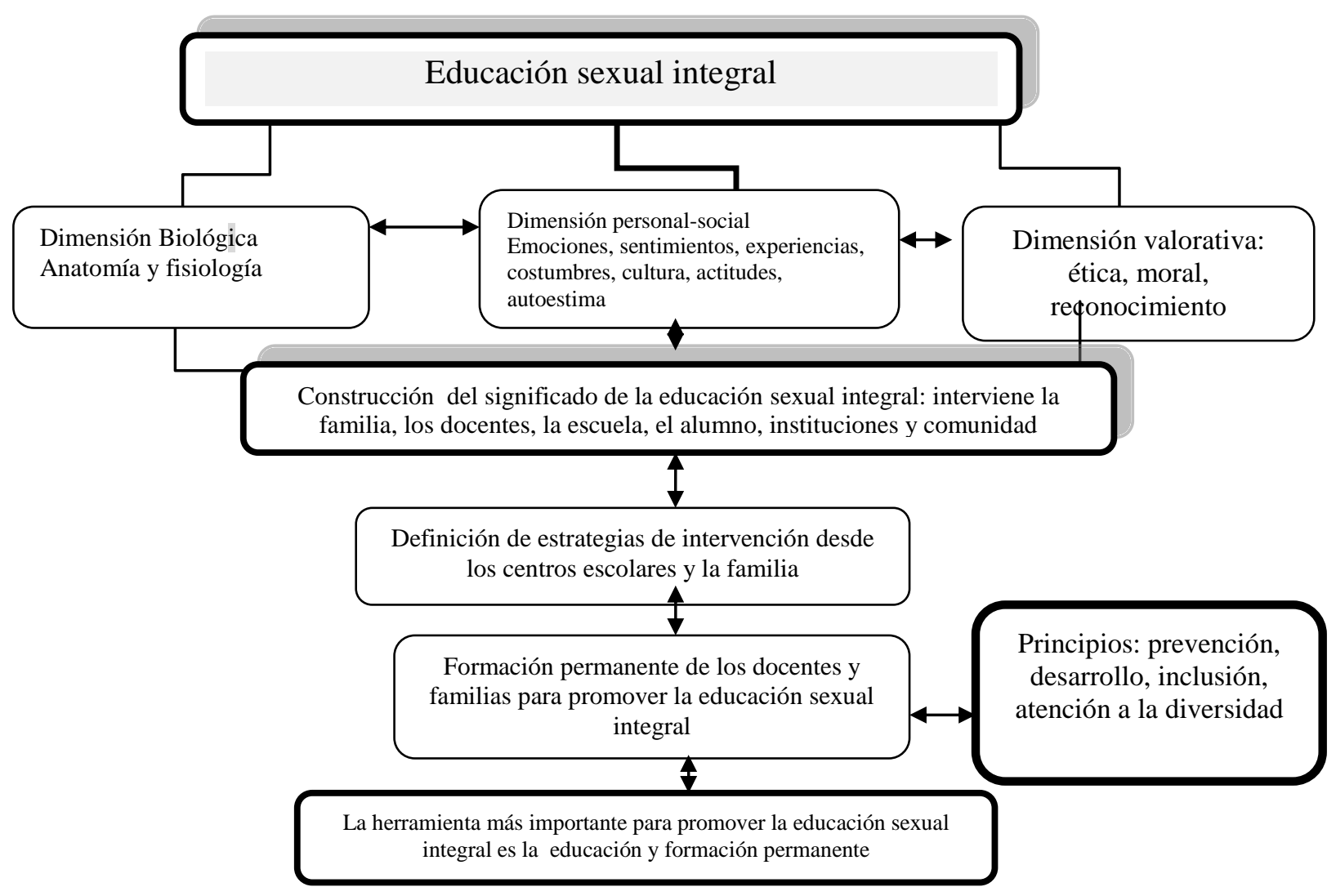

Figura 1: Elementos en el proceso de construcción del significado de la educación sexual

Los elementos expuestos en la figura anterior nos lleva a construir una aproximación a los lineamientos para promover en todos los espacios formativos escolares y extraescolares la educación sexual integral y contextualizada a la dinámica de los escolares en los centros educativos venezolanos.

\section{Método}

\section{Participantes}

El estudio se abordó en una muestra representada por 20 alumnos, 20 docentes y 15 familias pertenecientes al Grupo Escolar “ Guárico” y a la Unidad Educativa “Alto Barinas Norte" ubicado en el Municipio Barinas. Estado Barinas. Venezuela. La información fue recogida mediante registros diarios, entrevistas y cuestionarios con preguntas abiertas. Los datos fueron recogidos a lo largo de un año, mediante el uso de las técnicas e instrumentos señalados que han facilitado la organización, categorización y análisis de la información, para ello hicimos uso de la estadística descriptiva. 
La selección de los sujetos que integran la muestra se realizó mediante el criterio intencional o de propósito expuesto por Martínez (2007), este consiste en la selección de los sujetos significativos para el estudio en función de parámetros y criterios establecidos por el investigador. En efecto se escogieron los alumnos, docentes, y miembros de las familias que expresaron actitud positiva frente al estudio y se mostraron interesados en la temática.

\section{Enfoque y procedimiento}

La investigación inherente a la construcción del significado de la educación sexual en los centros escolares y el aula que promueve el docente en los centros escolares se abordó mediante una investigación descriptiva - etnográfica, donde se valoran los conceptos, significados, contenidos y prácticas que a lo largo de un determinado período de formación vienen asumiendo los alumnos, docentes y familias en los centros escolares y el aula.

La metodología que más correspondencia tiene con la finalidad de la investigación es la descriptiva- etnográfica. Mediante la descripción nos hemos podido apropiar de las características, rasgos y detalles de los ambientes escolares y familiares, así como, registrar las relaciones y comportamientos de los estudiantes: aspectos que se complementan con la etnografía definida por Goetz \& Le Compte (1988), como un método de investigación útil para conocer la cultura, relaciones, significados y experiencias que subyacen en los grupos. En tal sentido su utilidad en el estudio estuvo dirigida en conocer los conceptos y formas como describen la educación sexual.

Los estudios etnográficos exigen que el investigador se integre al grupo, a la comunidad y pueda vivenciar, captar, percibir el mundo de los significados desde adentro, implica aprender su lenguaje y costumbres, para hacer adecuadas interpretaciones de los sucesos, si se tienen en cuenta sus significados; no se trata de hacer una fotografía con los detalles externos, hay que ir más atrás y analizar los puntos de vista de los sujetos y las condiciones histórico-sociales en que se dan.

Es por eso que el etnógrafo tiene que insertarse en la vida del grupo y convivir con sus miembros por un tiempo prolongado, pues ante todo tiene la necesidad de ser aceptado en el 
grupo, después aprender su cultura, comprenderla y describir lo que sucede, las circunstancias en que suceden mediante el uso del mismo lenguaje de los participantes.

De allí que el principal medio para la apropiación de información y configuración de los significados que le otorgan los escolares, docentes, padres y familia a la educación sexual es mediante la etnografía constituyéndose en el quehacer como investigador y herramienta esencial del docente para el análisis y solución de problemas educativos. Así como se ha considerado un instrumento muy valioso en la investigación de campo para enriquecer la calidad del dato y ampliar o esclarecer la información.

\section{Instrumentos y técnicas}

Fundamentalmente se utilizó la observación participante mediante la cual se pudo captar, percibir y observar qué promueven los docentes con respecto a la educación sexual. Así mismo, se le otorgó importancia a la entrevista como técnica que permitió el contacto directo con los docentes, padres, representantes y alumnos. Ambas técnicas se apoyaron con el registro de campo, facilitando de esta manera la triangulación de la información.

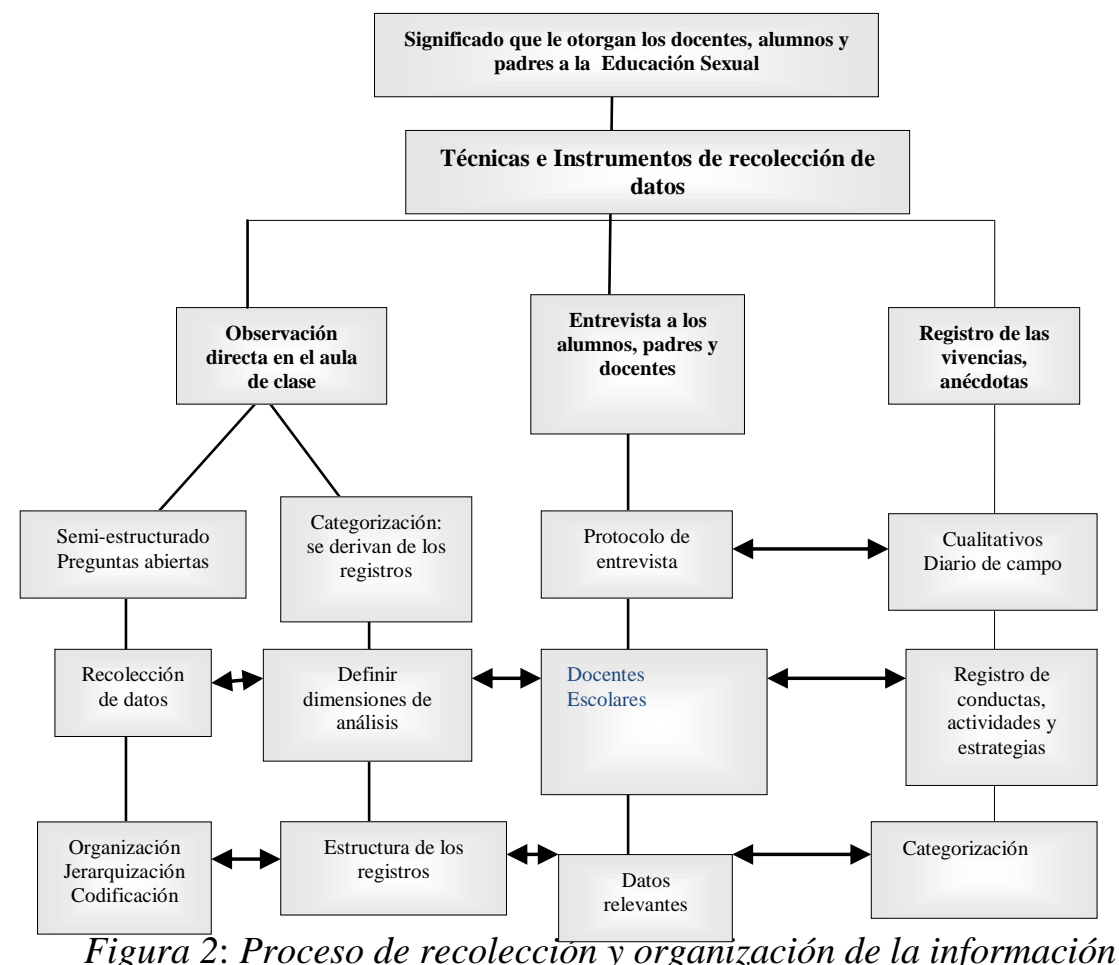

1) Observación directa en el aula 
Durante un año escolar se llevo a cabo la observación directa en las sección “A, B y C" de la Escuela Primaria "Guárico" ubicada en el centro de la ciudad del Municipio Barinas Estado Barinas, País Venezuela, correspondiente a escolares de 9, 10 y 11 años de edad, donde se vivenció el tipo de información, orientación y asesoría que brindan los docentes a los escolares con respecto a la educación sexual. También se llevaron registros de campo permanente de la actuación de los padres y representantes frente a la educación sexual de sus hijos y representados. La misma experiencia se abordó en las secciones 1, 2 y 3 de la escuela secundaria "Alto Barinas Norte", estos escolares tienen edades comprendidas entre 10, 11 y 12 años. Datos relevantes registrados durante un lapso escolar, los mismos inherentes a las actividades de educación y formación en educación sexual que promueve el centro escolar a nivel de los alumnos, la familia y la comunidad.

\title{
2) Formato de observación
}

Este registro se lleva diariamente, con el fin de determinar de qué manera el docente promueve la educación en el aula y valorar el nivel de participación de la familia.

Ver Cuadro 1.

\author{
Nombre del Docente \\ Fecha: \\ Hora:

\section{Actividades de inicio} \\ - Promoción de la educación sexual \\ - Tipo de información y/o formación promueve el docente en clase: enfermedades, riegos, fisiología \\ y anatomía
}

\author{
Actividades de desarrollo \\ Estrategias de formación \\ - Estrategias de formación en educación sexual promueve del docente? \\ - Participación de los alumnos en las actividades \\ - Integración de la familia \\ - Necesidades evidentes
}

\section{Actividades de cierre}

Actividades de retroalimentación

- Nivel afectivo en el aula

- Nivel de reforzamiento de las estrategias

- Grado de compromiso del docente con las actividades de educación sexual

- Relaciona el docente las experiencias a lo largo de la jornada de clase

\section{Otras actividades}

\section{3) Registros llevados a los padres}


La observación a las familias se llevo a cabo en los ambientes naturales, evidentemente sin que los alumnos fuesen conscientes de ello. La tarea de observación se realiza a lo largo del tiempo, dejando en todo momento debida nota de las observaciones. Una vez culminado el proceso de observación se procedió a comparar los resultados con los registros llevados a los docentes y alumnos. La plantilla para recoger los datos se presenta seguidamente:

- ¿Qué concepto manejan los padres de la educación sexual?

- ¿Qué valores promueven a nivel de los hijos e hijas y miembros de grupo familiar?

- ¿En que actividades de educación sexual le implica la escuela?

- ¿Con que frecuencia promueve la escuela la educación sexual?

- ¿Qué necesidades presentan las familias para promover la educación sexual?

\section{4) La Entrevista}

Kerlinger (1985) la entiende como....una confrontación interpersonal, en la cual una persona (el entrevistador) formula a otra (el respondiente) preguntas cuyo fin es conseguir contestaciones relacionadas con el problema de investigación. Según Galindo (1998), la entrevista es...una conversación que establecen un interrogador y un interrogado para un propósito expreso. (...) una forma de comunicación interpersonal orientada a la obtención de información sobre un objetivo definido.

La entrevista realizada a los alumnos, padres y representantes se caracterizó por ser no estructurada, se trabajó con preguntas abiertas, sin un orden preestablecido, adquiriendo características de conversación. Las preguntas se formulan teniendo como tema central el significado de la educación sexual para cada uno de ellos. Las respuestas fueron organizadas y categorizadas de acuerdo al nivel de ocurrencia, similitud, y significado otorgado a cada pregunta derivada de la conversación. Asumiendo esta conceptualización se utilizó la entrevista para recoger las vivencias, experiencias, actitudes, y datos relevantes inherentes a la educación sexual promovida por los docentes y la escuela a través de los proyectos de aula y educativos. Los padres y representantes tomados para el estudio corresponden a los padres de los escolares objeto de estudio durante un lapso escolar.

5) Registros de campo 
El registro de campo constituyó uno de los principales medios para plasmar las vivencias, experiencias, encuentros y desencuentros con los escolares, padres, madres y familia. Entre las principales dimensiones de registros se estudia la costumbre como práctica social arraigada que es. Generalmente se distingue entre buenas costumbres que son las que cuentan con aprobación social, y las malas costumbres, que son relativamente comunes, pero no cuentan con aprobación social. Los modos de actuar, de percibir la educación sexual tienen influencia directa y casi determinante de la costumbre, de los valores. De allí la importancia que tiene en la investigación la indagación en el aspecto sociocultural.

\section{Análisis de datos}

A partir de los registros llevados a lo largo del año escolar 2010 de los conceptos, vivencias, experiencias y prácticas formativas inherentes a la educación sexual, se han configurado resultados, organizados por categorías de análisis que han emergido de los propios actores de la investigación. Los datos se organizan de acuerdo a los objetivos del estudio. El análisis de los datos es el precedente para la actividad de interpretación.

La interpretación se realizó en términos de los resultados de la investigación. Esta actividad consiste en establecer inferencias sobre las relaciones entre las variables estudiadas para extraer conclusiones y recomendaciones (Kerlinger, 1985). La interpretación se realizó en dos etapas:

a) Interpretación de las relaciones entre las variables y los datos que las sustentan con fundamento en algún nivel de significancia estadística.

b) Establecer un significado más amplio de la investigación, es decir, determinar el grado de generalización de los resultados de la investigación.

Las dos etapas anteriores se sustentan el grado de validez y confiabilidad de la investigación. Ello implica la capacidad de generalización de los resultados obtenidos. “Analizar significa establecer categorías, ordenar, manipular y resumir los datos," (Kerlinger, 1985, p. 96). En esta etapa del proceso de investigación se procede a racionalizar los datos colectados a fin de explicar e interpretar las posibles relaciones que expresan las variables estudiadas. Los datos se presentan en tablas estadísticas que permitieron aplicar técnicas de 
análisis complejas facilitando este proceso. El análisis se expresa de manera clara y simple utilizando lógica tanto inductiva como deductiva.

Los resultados de la investigación basados en datos muestrales requieren de una aproximación al verdadero valor de la población (Zorrilla, 1994). Para lograr lo anterior fue necesaria una serie de técnicas estadísticas. Estas técnicas se derivan tanto de la estadística paramétrica como de la estadística no paramétrica. La primera tiene como supuestos que la población estudiada posee una distribución normal y que los datos obtenidos se midieron en una escala de intervalo y de razón. La segunda no establece supuestos acerca de la distribución de la población sin embargo requiere que las variables estudiadas se midan a nivel nominal u ordinal

\section{Resultados}

Una vez asumido el procedimiento anterior que implicó una revisión exhaustiva y depuración de los datos, con el fin de eliminar errores y omisiones posibles, se procedió a la codificación de cada categoría de respuesta obtenida de las técnicas e instrumentos aplicados, y la posterior tabulación y presentación de los resultados en una tabla de frecuencia.

Tabla $\mathrm{n}^{\circ}$ 1. Concepto de educación sexual que manejan los docentes

\begin{tabular}{c|l|c|c}
\hline Codificación & \multicolumn{1}{|c|}{ Categorías } & Frecuencia & Porcentaje \\
\hline 1 & $\begin{array}{l}\text { Información sobre la } \\
\text { prevención de } \\
\text { enfermedades sexuales }\end{array}$ & 10 & 50 \\
\hline 2 & $\begin{array}{l}\text { Conocimiento de los } \\
\text { órganos sexuales }\end{array}$ & 5 & 25 \\
\hline 3 & $\begin{array}{l}\text { Formación permanente en los } \\
\text { diferentes dimensión del } \\
\text { desarrollo humano }\end{array}$ & 5 & 100 \\
\hline Total & \multicolumn{1}{|c}{} & 20 docentes & \\
\hline
\end{tabular}

El 50\% de los docentes observados durante un año escolar expresan que la educación sexual consiste en la información sobre la prevención del embarazo precoz y de enfermedades sexuales, un $25 \%$ considera que el fin de la educación es la promoción del 
conocimiento de los órganos sexuales. Y un $25 \%$ expresan que consiste en la formación permanente en las diferentes dimensiones del desarrollo humano.

Los resultados expresados en la tabla anterior se sustentan en los discursos que hemos configurado de los registros de campo y entrevistas llevados a los docentes: La educación sexual se concibe en la escuela como un proceso de informaciòn permanente sobre la prevención del embarazo precoz y enfermedades sexuales, reduciendo a la educación sexual a un concepto reproductivo, asociado a enfermedades que atentan contra el desarrollo integral del escolar.

Tabla $n^{\circ}$ 2. Estrategias promovidas por el docente sobre la educación sexual

\begin{tabular}{c|l|c|c}
\hline Codificación & \multicolumn{1}{|c|}{ Categorías } & Frecuencia & Porcentaje \\
\hline 1 & $\begin{array}{l}\text { Desarrollo de programas de } \\
\text { intervención en educación sexual }\end{array}$ & 5 & 25 \\
\hline 2 & $\begin{array}{l}\text { Información mediante carteles, } \\
\text { carteleras, boletines y otros medios }\end{array}$ & 10 & 50 \\
\hline 3 & $\begin{array}{l}\text { Entrevistas con los escolares con } \\
\text { problemas sexuales }\end{array}$ & 5 & 25 \\
\hline Total & & 20 docentes & 100 \\
\hline
\end{tabular}

El $25 \%$ de los docentes promueven como estrategias el desarrollo de programas de intervención en educación sexual, un 50\% utiliza como estrategia la información mediante carteles, carteleras, boletines y otros medios de información: este tipo de estrategia es la que predomina fundamentalmente en los centros escolares como práctica de información ocasional inherente a la educación sexual. Y un $25 \%$ asume la entrevista como un medio para el abordaje de problemas sexuales.

La formación permanente del profesorado y de las familias se presenta como un mecanismo para modificar significados y prácticas que, según tal visión reduccionista, son construcciones obstaculizadoras que impiden el acceso efectivo a los derechos que se enuncian, referidos a la educación sexual integral. Causa preocupación el hecho de que muchos padres y docentes no están preparados para promover la educación sexual a los hijos y alumnos, porque ellos mismos nunca la tuvieron y porque sienten que el tema les sobrepasa sus responsabilidades y por lo tanto en la realidad, no se están haciendo cargo de esta responsabilidad social inherente a su rol como padres y formadores. 
En relación al significado que le otorgan los docentes a la educación sexual que se promueve en los centros escolares: el $25 \%$ de los docentes expresan que la educación sexual es un proceso de orientación que ayuda a los alumnos a conocerse a sí mismo como personas. Un 25\% de los docentes expresan que es la información que se brinda a los escolares para el conocimiento de su cuerpo humano, especialmente los órganos sexuales y un 50\% expresa que es la formación que reciben los alumnos en el hogar y en la escuela para llevar una vida sexual sana.

En cuanto a los planes y programas de educación sexual que se promueve en los centros escolares: el 20\% de los docentes expresan que se promueve la orientación educativa, un $30 \%$ la educación sexual se aborda mediante los proyectos de aula y plantel y un $40 \%$ sólo lo conciben como actividades de tipo informativas. Con respecto a las personas de las cuales los alumnos reciben directamente mayor información sobre la educación sexual encontramos por orden de importancia las siguientes: en primer lugar de sus compañeros de escuela y vecinos, en segundo lugar docentes y tercer lugar la familia.

Se plantea la necesidad de promover una mayor participación de los padres, junto con los docentes en las actividades de educación sexual que planifica la escuela, se trata de procurar una visión compartida y dialógica. La escuela debe trabajar para motivar e integrar los padres a la escuela y asumir la educación sexual como un eje transversal que permee todos los actos d formativos de los escolares. La formación permanente de los padres contribuiría al cambio de actitud frente a los temas de educación sexual y les abriría una nueva opción para conducir e interpretar el rol como agentes educativos y de prevención integral.

De acuerdo a lo expresado por algunos escolares, los padres no hablan abiertamente de la educación sexual con sus hijos, sienten temor, tienen miedo, actitud asumida en la mayoría de los casos por sus experiencias, vivencias y significados culturales. Un estudio realizado por Rogow \& Haberland (2005) demuestran que un programa efectivo debería tener la capacidad de: reducir la información incorrecta; aumentar el conocimiento y manejo de información correcta; consolidar valores y actitudes positivas; fortalecer las competencias necesarias para toma decisiones fundamentadas y la capacidad de actuar en función de ellas; mejorar las percepciones acerca de los grupos de pares y las normas sociales; y aumentar y mejorar la comunicación con padres madres y otros adultos de confianza.

Para los autores arriba mencionados puede resultar valioso reenfocar los programas y dar menos énfasis a los aspectos puramente biológicos de la sexualidad 
para hacer mayor hincapié, sobre todo al principio, en el contexto social en el que se forman las actitudes sexuales, se toman las decisiones y se interpretan los papeles sexuales. Esta reorientación de enfoque debería situarse no exclusivamente en un contexto de salud/biología, sino como parte de lo que se podría llamar 'estudios sociales' o 'educación ciudadana.'

También se configuraron las siguientes categorías definidas como necesidades de los escolares que reflejan la limitada educación sexual que reciben en los centros de formación en los primeros años de escolarización.

Tabla 3. Necesidades que se identificaron en los alumnos

\begin{tabular}{c|l}
\hline Codificación & \multicolumn{1}{c}{ Categorías } \\
\hline 1 & Nula información sobre la sexualidad humana \\
\hline 2 & $\begin{array}{l}\text { Temor, vergüenza y miedo para tratar abiertamente los temas de sexualidad } \\
\text { en la escuela }\end{array}$ \\
\hline 3 & $\begin{array}{l}\text { Información que reciben en la escuela está centrada en las enfermedades y riesgos } \\
\text { de contraer enfermedades sexuales }\end{array}$ \\
\hline 4 & Ausencia de programas de educación sexual integral integrados al acto formativo \\
\hline 5 & $\begin{array}{l}\text { Escasa orientación personal social dirigido a los escolares a fin de esclarecer sus } \\
\text { inquietudes }\end{array}$ \\
\hline 6 & Conocimiento sexual basados en creencias, prejuicios y estereotipos \\
\hline
\end{tabular}

Se determinó a nivel de los alumnos la limitada o nula información sobre la sexualidad humana, así como el manifiesto temor, vergüenza y miedo para comunicar abiertamente los temas inherentes a la educación sexual. La orientación y ayuda que le ofrece la escuela está centrada en la información sobre las enfermedades y riesgos, es decir en las consecuencias, dejando claramente denotado un concepto de represión de la sexualidad. A juicio de los escolares la escuela no promueve programas de educación sexual integrados al acto formativo, aunada a la limitada orientación personal social que ayuden a los alumnos a esclarecer sus inquietudes y expectativas frente a la sexualidad.

En cuanto al concepto que manejan los alumnos sobre la educación sexual: existe en los resultados la evidente tendencia entre más del $50 \%$ de los escolares en conceptualizar la educación sexual como un medio para el conocimiento de los órganos sexuales, configurándose una visión sesgada y reducida de la amplitud del concepto de educación 
sexual integral que debemos manejar, asumida como "el proceso de orientación dirigida a desarrollar e intervenir en la salud sexual de los niños, niñas, adolescentes, adultos y personas en general, favoreciendo la salud sexual en los aspectos físicos, emocionales, intelectuales y sociales relativos a la sexualidad, para promover el bienestar personal y social mediante la comunicación permanente bidireccional y humana" .

A juicio de los escolares de primaria: “... en mi casa y en la escuela pocas veces se habla de la educación sexual, en varias oportunidades le pregunte a mi mamá como nació mi hermana y me dijo eso no se pregunta, lo poco que sé de mi cuerpo es por un amigo que me dice, como a medida que uno crece se va desarrollando..."

“... en la escuela la maestra nos ha enseñado las partes del cuerpo humano, pero pocas veces ha conversado con nosotros sobre la educación sexual...”

“... la escuela debe abordar la educación sexual desde la prevención, en la escuela se habló de embarazo precoz cuando María mi compañera de estudio salido en estado, ya para que..."

Con respecto a las estrategias promovidas por el docente sobre la educación sexual: El 50\% de los escolares consideran que los docentes intervienen con charlas, cursos, talleres, exposiciones, y conversaciones en el aula de clase, un $25 \%$ de los docentes promueven solo carteleras informativas y un $25 \%$ expresan que la educación sexual se aborda en la hora de guiatura.

Tal como se evidencia en los registros la escuela fundamentalmente viene asumiendo la información más que la formación en educación sexual, las acciones están centradas al cumplimiento de determinados objetivos curriculares, en la mayoría de los casos de dominio cognitivo. A juicio de un escolar de primaria"... mi participación en la escuela se ha limitado solo a la elaboración de carteleras informativas inherentes a los órganos reproductores femeninos y masculinos..."

Se denota en esta experiencia un concepto de sexualidad desde el punto de vista reproductivo: aprendizaje de la función de los órganos sexuales.

Así mismo se han configurado categorías de análisis derivados de los registros de campo llevados a las familias sometidas a estudio entre las que se citan en la tabla 4 las necesidades manifiestas por las familias en materia de educación sexual. 
Tabla 4.Necesidades que se identificaron en las familias

\begin{tabular}{c|l}
\hline Codificación & \multicolumn{1}{c}{ Categorías } \\
\hline 1 & $\begin{array}{l}\text { Los padres expresan que no han recibido la formación necesaria para } \\
\text { orientar y ayudar a los hijos e hijas en temas de educación sexual }\end{array}$ \\
\hline 2 & $\begin{array}{l}\text { Manifiestan limitada comunicación y confianza entre los padres e hijos, } \\
\text { para conversar sobre la sexualidad }\end{array}$ \\
\hline 3 & $\begin{array}{l}\text { Asumen que la escuela es la responsable de impartir solo información en } \\
\text { educación sexual }\end{array}$ \\
\hline 4 & $\begin{array}{l}\text { Escasas veces se implican en actividades formativas de educación sexual } \\
\text { promovidas por los centros escolares. }\end{array}$ \\
\hline
\end{tabular}

Las familias a quienes se les llevó registros permanentes de las actitudes, conceptos y significados de la educación sexual expresan: “... la limitada formación para la orientación a sus hijos e hijas en esa materia, así como expresan que no existe un nivel confianza y seguridad para conversar y orientar abiertamente a sus hijos e hijas y miembros del grupo familiar. Opinan que la escuela no les ha brindado la suficiente información y capacitación con respecto al tema..."

Para un grupo de familias la educación sexual es un acto de cada persona: “... nosotros aprendimos en la escuela de la vida...". De allí que los comportamientos sexuales: muchas veces, se basan sobre creencias erróneas prejuicios o estereotipos de género. Los padres consideran que no se sienten capacitados para orientar en temas de sexualidad a sus hijos e hijas.

Los padres y representantes, e incluso la familia, son de acuerdo a los resultados los más desvinculados de la escuela en cuanto a su intervención en la formación de los escolares en educación sexual: para la mayoría de los padres, el centro escolar pocas veces aborda la temática de la sexualidad, su rol esta más centrado en el dominio de los contenidos y en el cumplimiento de normas institucionales.

Para los padres es preocupante que cada vez más niñas y niños se vean implicados en actividades sexuales irresponsables. Creemos que la escuela debe desarrollar en los escolares el conocimiento de sí mismo, fortalecer la autoestima, orientar a los padres y madres que tienen conflictos familiares, guiar y apoyar a los escolares con familias disfuncionales; educados por la madre, el padre o un familiar. 


\section{Conclusiones y recomendaciones}

De los resultados obtenidos producto de la aplicación de técnicas e instrumentos se han obtenido las siguientes conclusiones, según cada uno de los objetivos de investigación:

En cuanto a las estrategias de promoción de la educación sexual en los centros escolares y el aula se concluye que más del $50 \%$ de los estudiantes consideran que fundamentalmente se promueve la información sobre temas inherentes a la educación sexual, en correspondencia con la mayoría de los casos la visión que se tiene de la sexualidad es reduccionista y estrecha, con frecuencia se piensa en los genitales y la relación física entre las personas, la mayoría de los programas educativos giran en torno a la llamada reproducción y en todo caso como prevenir un embarazo, como si fuese el único aspecto de la sexualidad..."

En cuanto al significado que le otorgan los docentes, alumnos y comunidad en general a la educación sexual en el marco de la diversidad, un alto porcentaje de los escolares consideran que el significado otorgado, tiene correspondencia con la cultura, costumbres, opiniones y experiencias de cada una de las personas, reduciendo la educación sexual a una determinada dimensión del desarrollo humano, desvinculado de la visión de integralidad que debe prevalecer en la concepción de la educación sexual.

De la investigación se derivan las siguientes categorías que le otorgan significado a la educación sexual: información inherente al funcionamiento de los órganos sexuales; formación de valores de responsabilidad y ética como seres sexuales; conocimiento del cuerpo humano y sus órganos reproductores y; valoración psíquica y social como hombres y mujeres que necesitan ser amados, queridos y respetados.

En cuanto a las políticas, programas, servicios y condiciones para promover la educación sexual integral en el marco de la diversidad se determinó: que se le otorga un amplio tratamiento teórico a nivel de políticas a la educación sexual tanto en los tratados internacionales, con en los planes, programas y proyectos a nivel nacional e internacional. Así como se evidencia una desvinculación entre la teoría y la practica de la educación sexual en los centros escolares y el aula.

\section{Recomendaciones}


La revisión teórica inherente a la educación sexual y la indagación producto de la investigación descriptiva y etnográfica ha permitido perfilar los siguientes lineamientos que ayudarían al abordaje de la educación sexual integral desde las políticas educativas hasta integrarlos a los currículos de la educación inicial y primaria, hasta la universidad y después de egresados, es decir a lo largo de toda la vida de la persona.

Definir desde el Ministerio de Educación del País, políticas de educación sexual que sean integrados a los proyectos educativos bajo la concepción valorativa del contexto familiar y comunitario donde está inmerso el escolar, otorgándole importancia durante el proceso formativo a las experiencias, vivencias, cultura, tradiciones y conocimientos que le han servido al escolar para construir su propio concepto de educación sexual.

Así mismo debe concebirse en los proyectos educativos de formación como un derecho inherente al ser humano individual y social, donde el compromiso es de todos: docentes, familia, comunidad, instituciones y estado.

Los centros escolares han de formular planes y proyectos que les conecten con las instituciones de la comunidad, incluyendo los medios de comunicación, preferentemente la radio y la televisión por el potencial para comunicar e informar sobre la prevención y desarrollo de programas dirigidos a concienciar y socializar a la comunidad en cuanto a temas inherentes a la educación sexual.

La educación sexual debe asumirse desde un enfoque integral que considera a la persona como un ser biológico, físico, psíquico y social: lo que implica fomentar el cuidado y la responsabilidad en el ejercicio de la sexualidad, promoviendo la paternidad/maternidad responsable y la prevención de las enfermedades de transmisión sexual y promover la modificación de los patrones socioculturales estereotipados con el objeto de eliminar prácticas basadas en el prejuicio de superioridad de cualquiera de los géneros.

\section{Referencias}

Asamblea Nacional (1999). Constitución de la República Bolivariana de Venezuela. Gaceta oficial $\mathrm{N}^{\mathrm{o}} 36.860$. 
Asamblea Nacional (2007). Ley de personas con discapacidad. Gaceta oficial de la República Bolivariana de Venezuela $\mathrm{N}^{\circ} 38598$ (extraordinario).

Asamblea Nacional (2009). Ley orgánica de educación. Gaceta oficial de la República Bolivariana de Venezuela $\mathrm{N}^{\circ} 5929$ (extraordinario).

Cook, T. \& Reichardt, Ch. (1997). Métodos cualitativos y cuantitativos en investigación educativa. Madrid: Morata.

Clement, A. (2007) Educación sexual en la escuela. Perspectivas y reflexiones. Buenos Aires: Gobierno de la Ciudad de Buenos Aires.

De Luca, C (2009) Implicaciones de la Formación en la autoestima del estudiante universitario. Electronic Journal of Research in Educational Psychology, 7(2), 901922.

Deborah, R \& Nicole, H (2005). Educación sobre sexualidad y relaciones: hacia una perspectiva de estudios sociales. Population Council, New York, USA Sex Education Vol. 5, No. 4.

Echeita, G. (2005). En el camino hacia una educación más inclusiva. El Index for. Inclusión. Temáticos Escuela.

Flórez, G, Lugo, A \& Moya, D (2009). Conocimientos generales sobre sexualidad en adolescentes. Área urbana de la ciudad de Punto Fijo Residentes del Hospital Pediátrico Dr. García Coello. Venezuela.

García. J. \& Juan F. (1992). Práctica anticonceptiva en adolescentes y jóvenes. Área metropolitana de la ciudad de México. Julio- Agosto. Vol. 34 N0. 4.

Galindo, M (1998). Técnicas de investigación en sociedad, cultura y comunicación. Compilación. Addison Wesley Longman. México. 345

Goetz JP \& Le Compte. (1988). Etnografía y diseño cualitativo en investigación educativa. MD. Madrid: Morata.

Hernández, F \& Baptista, P. (2004). Metodología de la investigación. México: McGraw-Hill.

Hiriart, V (2000). Educación Sexual en la Escuela. Guía para el orientador de púberes y adolescentes. Paidós 
Jaímes, R (1983). Federación Internacional de Planificación de la Familia. "Sexualidad humana y relaciones personales.

Kerlinger, F (1982). Enfoque conceptual de la investigación del comportamiento. México: Interamericana.

López, B., Mancias, T. y Col. (1995). Patrones de Crecimiento de los Venezolanos. Dimosfismo Sexual y Ritmo de Maduración. Archivos Venezolanos de Pediatría, Vol. 58. N 4.Octubre>Noviembre, pág. 163-170.

Martínez, M. (2007). Investigación cualitativa. Etnografía en educación. Manual Teórico Práctico. México: Trillas.

Molina, D. y De Luca, C (2009). Orientación Integral en los Centros Educativos y en el aula. Electronic Journal of Research in Educational Psychology, 7(3), 1449-1460.

Medrano, H. (2001). Atención a la diversidad desde la calidad y la equidad en la educación básica. Tesis Doctoral. España: Universidad Autónoma de Barcelona. Recuperado el 26 de noviembre de 2008 de: http://www.tesisenxarxa.net/TDX-1026101$\underline{104924 / \text { indexcs.html }}$

Méndez, D y Beltrán, A (1991). II Congreso Venezolano de la Mujer. Caracas

Ministerio de Educación Superior (2004). Derecho de las Personas con discapacidad a una educación superior de calidad. Caracas: Ministerio de Educación.

Ministerio del Poder Popular para la Educación Superior (2008). Reglamento Orgánico. Gaceta Oficial de la República Bolivariana de Venezuela, № 39032.

Molina, D. L. (2009). Hacia una educación integral. Los elementos clave en la escuela de la vida. Barcelona: Erasmus Ediciones.

Organización de Estados Americanos (1999). Convención Interamericana para la eliminación de todas las formas de discriminación contra las personas con discapacidad. Aprobada en asamblea general por el consejo permanente en sesión. Guatemala: OEA. 
Organización de Estados Iberoamericanos para la Educación, la Ciencia y la Cultura (1990). Declaración mundial sobre educación para todos. Satisfacción de las Necesidades Básicas de Aprendizaje. Tailandia: OEI.

Organización de las Naciones Unidas para la Educación, la Ciencia y la Cultura (2000). Declaración de Salamanca. Conferencia Mundial sobre Necesidades Educativas Especiales: acceso y calidad. Salamanca: UNESCO.

Sáez, G. (1992). La salud del adolescente y la acción en la comunidad. 2da Edición, UNICEF, Vol. N 1. Julio. Venezuela. pág. 7-10.

Sandín, E. (2003). Investigación cualitativa en educación. Fundamentos y tradiciones. Madrid: Mcgraw-Hill.

Sierra, R. (1998). Tesis doctorales y trabajos de investigación científica. Madrid: Paraninfo.

Papalia, D., Wendkos, A. y Duskin, R. (2001). Desarrollo Humano. Bogotá: Editorial Mc Graw-Hill. Interamericana, S. A.

Papalía, E. (2002). Sexualidad Humana. Mc Graw-Hill. Interamericana, S. A.

Rogow, D \& Nicole H. (2005). "Sexuality and relationships education: Toward a social studies approach," Sex Education 5(4): 333-344. Also available in Spanish and French.

Zorrilla, S (1994). Introducción a la metodología de la investigación. Ediciones Océano. México. 